\title{
Aplicabilidade do Método da Transformada Diferencial na Modelagem Fracionária
}

\author{
Lucas Kenjy Bazaglia Kuroda ${ }^{1}$ \\ Departamento de Bioestatística, UNESP/IBB, Botucatu, SP \\ Alexys Bruno Alfonso ${ }^{2}$ \\ Departamento de Matemática, UNESP/FC, Bauru, SP \\ Rubens de Figueiredo Camargo ${ }^{3}$ \\ Departamento de Matemática, UNESP/FC, Bauru, SP \\ Paulo Fernando de Arruda Mancera ${ }^{4}$ \\ Departamento de Bioestatística, UNESP/IBB, Botucatu, SP
}

Resumo. Com o intuito de resolver equações diferenciais de ordem fracionária, utilizamos o método computacional conhecido como "Multi-step Generalized Differential Transform Method"(MSGDTM) que utiliza a forma de polinômios como aproximações das soluções. Nota-se que apesar deste método ser muito utilizado por alguns autores, existem alguns pontos a serem discutidos sobre sua aplicabilidade em modelos de equações de ordem nãointeiras, dentre eles destacamos questões associadas com a não localidade da derivada fracionária.

Palavras-chave. Cálculo Fracionário, Método Numérico, MSGDTM, Modelagem Fracionária, Método da Transformada Diferencial.

\section{Introdução}

A Modelagem Matemática é um processo que consiste em traduzir uma situação ou tema do meio em que vivemos para uma linguagem matemática. São conduzidos ao problema de se determinar uma função a partir do conhecimento prévio de suas taxas de variação, isto é, uma equação diferencial. Esta descrição compreende as etapas de identificação das variáveis do problema e de um conjunto de hipóteses razoáveis sobre o sistema [12]. Assim, quanto mais complexa essa equação for, maior será a dificuldade em encontrar sua solução.

A obtenção de uma equação diferencial cuja solução descreve bem a realidade traz grande dificuldade. Normalmente, quanto mais próximos estamos de descrever um problema real, maior será o número de variáveis envolvidas ea complexidade das equações.

\footnotetext{
${ }^{1}$ lucaskuroda@ibb.unesp.br

2 alexys@fc.unesp.br

${ }^{3}$ rubens@fc.unesp.br

${ }^{4}$ pmancera@ibb.unesp.br
} 
Nesse sentido, o Cálculo de Ordens Não Inteira, tradicionalmente conhecido como Cálculo Fracionário, que é o ramo da matemática que estuda integrais e derivadas de ordens nãointeiras, desempenha este papel em destaque [3,9].

Considerando uma equação diferencial que descreve um fenômeno específico, uma maneira comum de usar a modelagem fracionária é substituir as derivadas de ordem inteira por uma derivada não-inteira, geralmente com ordem menor ou igual à ordem das derivadas originais, de modo que a derivada usual pode ser recuperada como um caso particular [9]. Assim, o principal desafio passa ser em buscar técnicas e métodos que encontre a solução da equação de estudo. Por exemplo, se tratando de equações lineares de ordem fracionárias, podemos utilizar a metodologia da transformada de Laplace. Note que existe uma grande ilimitação na utilizacão desta metodologia, uma vez existe uma ramo enorme de equações são não lineares, há também casos envolvendo sistema de equações, etc. Nesse caso, muitos autores utilizam técnicas computacionais para encontrar as soluções destas equações, dentre elas está o conhecido método "Multi-step Generalized Differential Transform Method"(MSGDTM) que utiliza a forma de polinômios como aproximações das soluções.

O método MSGDTM foi utilizado por alguns autores para encontrar soluções aproximadas do modelo de abandono do tabagismo, neste trabalho fo realizado um estudo comparativo entre o MSGDTM e o método de Runge-Kutta clássico no caso de derivadas de ordem inteira [5], o mesmo foi desenvolvido no modelo epidemiológico modificado para vírus de computador de [7], na solução de co-infecção por HIV e malária [4] e no modelo de dinâmica tumoral [2]. Nas soluções do sistema Chua, descobriram que o sistema de ordem fracionária com "dimensão efetiva" inferior a três pode apresentar caos assim como outros comportamentos não-lineares. Citam que o método tem a vantagem de dar uma forma analítica da solução dentro de cada intervalo de tempo que não é possível utilizando técnicas puramente numéricas como o método Runge-Kutta de quarta ordem [6].

Veremos a seguir que este método (MSGDTM) também possui limitações, uma vez que, embora não exista uma interpretação física e geométrica trivial para a derivada e a integral fracionária, as equações diferenciais de ordem fracionária estão naturalmente relacionadas a sistemas com memória, as derivadas fracionárias normalmente não são operadores locais, isto é, o cálculo da derivada depende de tempo anteriores [9]. Assim, será realizada uma comparação entre a solução analítica da equação de Malthus de ordem inteira e não-inteira com o método MSGDTM com um passo, dois passos, quatro passos e oitenta passos.

\section{2 "Multi-step Generalized Differential Transform Method"}

O método da transformada diferencial (DTM) é um método analítico e numérico para resolver uma grande variedade de equações diferenciais e fornece a solução em forma de série. Neste trabalho vamos apresentar um novo algoritmo adequado de DTM, ou seja, Multi-step Differential Transform Method (MSDTM), que aumentará o intervalo de convergência para a solução da série. O MSDTM é tratado como um algoritmo em uma sequência de intervalos para encontrar soluções precisas aproximadas para sistemas de equações diferenciais $[8,11]$. 


\subsection{Differential Transform Method (DTM)}

A técnica da transformada diferencial utiliza a forma de polinômios como aproximações das soluções exatas de equações diferenciais ordinárias e parciais. A transformada diferencial, $F(k)$, da $k$-ésima derivada da função $f(t)$ é definida como [11],

$$
F(k)=\frac{1}{k !}\left[\frac{d^{k} f(t)}{d t^{k}}\right]_{t=t_{0}},
$$

Já a transformada inversa de $F(t)$ é definida da seguinte maneira:

$$
f(t)=\sum_{k=0}^{\infty} F(k)\left(t-t_{0}\right)^{k} .
$$

De acordo com as equações (1) e (2), temos

$$
f(t)=\sum_{k=0}^{\infty} \frac{\left(t-t_{0}\right)^{k}}{k !}\left[\frac{d^{k} f(t)}{d t^{k}}\right]_{t=t_{0}},
$$

Assim, a transformada diferencial fornece os coeficientes da expansão em série de Taylor. No entanto, as correspondentes derivadas são calculadas iterativamente pelas equações transformadas da função original. Para fins de implementação, a função $f$ é expressa por uma série finita. Assim, a equação (2) pode ser escrita como [1,10,11],

$$
f(t) \approx \sum_{k=0}^{S} F(k)\left(t-t_{0}\right)^{k},
$$

em que $S$ é suficientemente grande. Para resolver os problemas não-lineares utilizando o DTM, precisamos primeiramente aplicar a transformação diferencial (1) na equação de estudo, resultando em uma relação de recorrência, depois resolvendo esta equação utilizando a transformada diferencial inversa (2), obtemos a solução do problema [8].

$\mathrm{Na}$ Tabela 1, apresentamos algumas propriedades da transformada diferencial para $t_{0}=0$.

Na próxima seção, será inserida a deriavada de ordem não-inteira nas equação envolvendo o método DTM. Os conceito preliminares do cálculo fracionário podem sem visto em [3].

\subsection{Generalized Differential Transform Method (GDTM)}

Consideremos uma função denotada por $f\left(t, u_{1}, u_{2}, \ldots, u_{n}\right)$, sua transformada diferencial será da forma $F\left(k, U_{1}, U_{2}, \ldots, U_{n}\right)$. Para encontrar uma solução de um sistema de equações diferenciais fracionárias com derivada de Caputo, dada pela equação de ordem $\beta_{i} \in(0,1]$, vamos utilizar o método da transformada diferencial generalizada, GDTM. Para isso, consideremos o sistema de equações diferenciais fracionárias sujeito às condições iniciais $u_{i}\left(t_{0}\right)=\lambda_{i}$ para $i=1,2, \ldots, n,[8]$ 
Tabela 1: Propriedades da Transformada Diferencial.

\begin{tabular}{ll}
\hline Função Original & Função Transformada \\
\hline$f(t)=\alpha x(t)$ & $F(k)=\alpha X(k)$ \\
$f(t)=x(t) \pm y(t)$ & $F(k)=X(k) \pm Y(k)$ \\
$f(t)=x(t) y(t)$ & $F(k)=\sum_{l=0}^{k} X(l) Y(k-l)$ \\
$f(t)=\frac{d}{d t} x(t)$ & $F(k)=(k+1) X(k+1)$ \\
$f(t)=\frac{d^{m}}{d t^{m}} x(t)$ & $F(k)=(k+1)(k+2) \ldots(k+m) X(k+m)$
\end{tabular}

$$
\left\{\begin{array}{c}
D^{\beta_{1}} u_{1}(t)=f_{1}\left(t, u_{1}, u_{2}, \ldots, u_{n}\right) \\
D^{\beta_{2}} u_{2}(t)=f_{2}\left(t, u_{1}, u_{2}, \ldots, u_{n}\right) \\
\vdots \\
D^{\beta_{n}} u_{n}(t)=f_{n}\left(t, u_{1}, u_{2}, \ldots, u_{n}\right)
\end{array}\right.
$$

Sendo $\left[t_{0}, T\right]$ o intervalo de busca de solução do problema de valor inicial, utilizando o GDTM (Generalized Differential Transform Method), a solução aproximada para a $S$ ésima ordem é ${ }^{5}$,

$$
u_{i}(t)=\sum_{k=0}^{S} U_{i}(k)\left(t-t_{0}\right)^{k \beta_{i}}, \quad t \in\left[t_{0}, T\right],
$$

em que cada $U_{i}$ satisfaz a seguinte relação,

$$
\frac{\Gamma\left[(k+1) \beta_{i}+1\right]}{\Gamma\left(k \beta_{i}+1\right)} U_{i}(k+1)=F_{i}\left(k, U_{1}, U_{2}, \ldots, U_{n}\right),
$$

sendo $U_{i}(0)=\lambda_{i}$.

\subsection{Multi-step Generalized Differential Transform Method (MSGDTM)}

Com base no trabalho de [2], nessa seção discutiremos o método conhecido como "Multi-step generalized differential transform method"(MSGDTM). Consiste em dividir o intervalo $\left[t_{0}, T\right]$ em $M$ subintervalos $\left[t_{m-1}, t_{m}\right], m=1,2, \ldots, M$, de tamanho $h=\frac{T-t_{0}}{M}$.

\footnotetext{
${ }^{5}$ Tomando a ordem da derivada fracionária igual a 1, podemos encontrar a solução da equação (4), ou seja, como o próprio nome diz, o método GDTM é uma generalização do DTM, o primeiro aplicado em equações de ordem fracionária e o segundo para equações de ordem inteira.
} 
Assim, obtemos como solução

$$
u_{i}(t)=\left\{\begin{array}{cc}
u_{i, 1}(t) & \text { se } t \in\left[t_{0}, t_{1}\right], \\
u_{i, 2}(t) & \text { se } t \in\left[t_{1}, t_{2}\right], \\
\vdots & \\
u_{i, M}(t) & \text { se } t \in\left[t_{M-1}, t_{M}\right],
\end{array}\right.
$$

sendo $u_{(i, 1)}(t)$ a solução aproximada do problema de valor inicial (5) aplicando o GDTM no intervalo $\left[t_{0}, t_{1}\right]$ com a condição inicial $u_{i}\left(t_{0}\right)=\lambda_{i}$. Para $m \geq 2$, aplicamos o GDTM no intervalo de $\left[t_{m-1}, t_{m}\right]$ usando a condição inicial $u_{i, m}\left(t_{m-1}\right)=u_{i, m-1}\left(t_{m-1}\right)$.

\section{Modelo de Malthus}

O modelo apresentado por Malthus, é utilizado na modelagem de crescimento de populacional, em que a taxa segundo qual a população cresce em um determinado instante é proporcional a população naquele instante. Matematicamente [3],

$$
\frac{d N(t)}{d t}=r N(t)
$$

sendo $N(t)$ a popualção no instante $t$ e $r>0$ uma constante de proporcionalidade.

\subsection{Modelagem Fracionária}

A partir do modelo de Malthus na equação(9), iremos utilizar a derivada fracionária segundo Caputo nesta equação, sendo $0<\beta \leq 1$ a ordem da derivada. Assim,

$$
D^{\beta} N(t)=\frac{d^{\beta} N(t)}{d t^{\beta}}=r N(t) .
$$

Através da metodologia da transformada de Laplace, temos que a solução analítica deste modelo $[3,8]$,

$$
N(t)=N(0) E_{\beta}\left(r t^{\beta}\right) .
$$

sendo $E_{\beta}\left(r t^{\beta}\right)$ a função de Mittag-Leffler [3].

A seguir, iremos comparar as soluções analíticas com as computacionais utilizando o método MSGDTM, sendo $r=1$ e $N(0)=1$.

Pelas Figuras 1 e 2, nota-se que ao utilizar o método MSGDTM com apenas um passo, GDTM, a solução numérica se aproxima da solução analítica. Quando acrescentamos alguns passos, a solução numérica sofre um crescimento elevado para cada passo do método, uma vez que o MSGDTM está tratando a derivada fracionária como uma operador local. Ou seja, para cada passo do método, os limites de integração referentes a derivada fracionária de Caputo são alterados, havendo esse crescimento inesperado. Quanto mais passos o método tiver, mais a curva de solução será suavizada, não sendo perceptível esses picos de crescimento. 

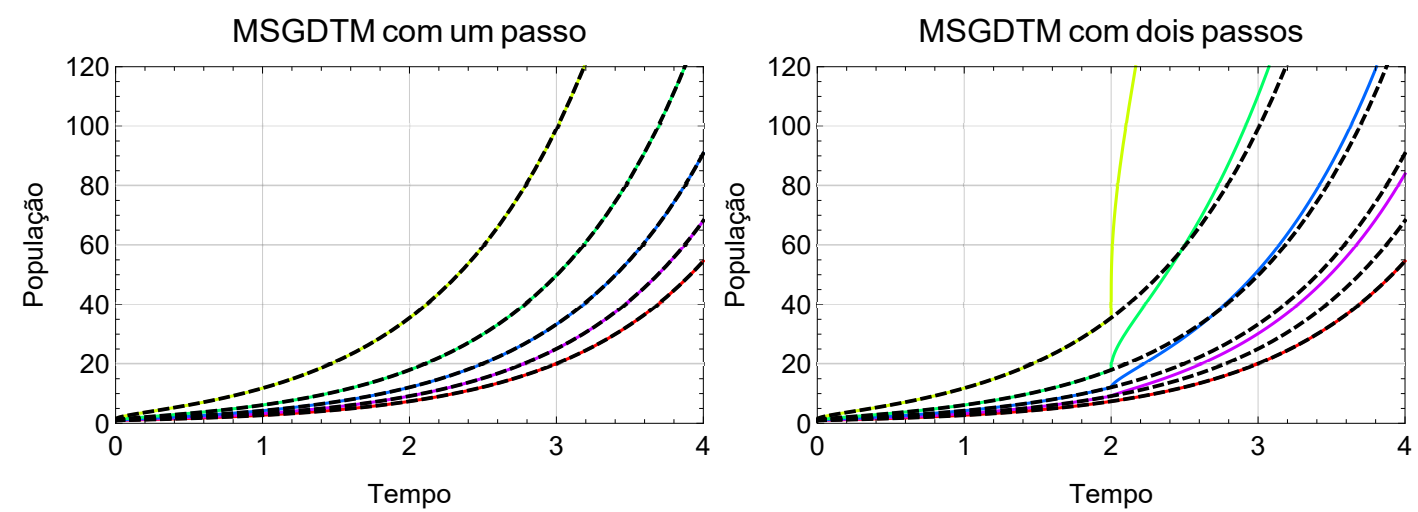

Figura 1: Método MSGDTM vs. Solução analítica: As linhas tracejadas correspondem a solução analítica, enquanto as linhas contínuas correspondem ao método MSGDTM para $\beta=1, \beta=0.8$, $\beta=0.6, \beta=0.4$ e $\beta=0.2$ respectivamente de baixo para cima.
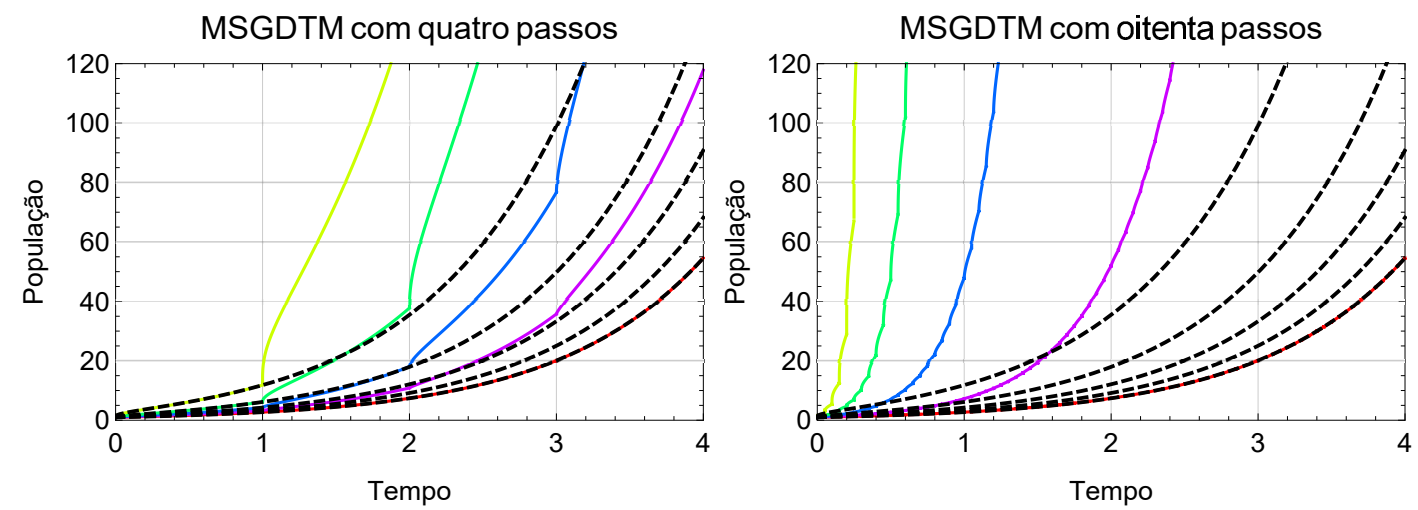

Figura 2: Método MSGDTM vs. Solução analítica: As linhas tracejadas correspondem a solução analítica, enquanto as linhas contínuas correspondem ao método MSGDTM para $\beta=1, \beta=0.8$, $\beta=0.6, \beta=0.4$ e $\beta=0.2$ respectivamente de baixo para cima.

\section{Conclusões}

A modelagem fracionária possibilita, em alguns casos, descrever um fenômeno de maniera mais realista. No entanto, algumas equações e sistemas de equações ainda não possuem métodos analíticos de solução. Por isso, muitos autores utilizam técnicas computacionais para resolver esses problemas. Um dos métodos utilizados é o MSGDTM, no entanto, notamos que este método trata a derivada fracionária como um operador local, uma vez que o limite de integração da definição da derivada de Caputo é alterada, havendo um crescimento inesperando para cada passo do "Multi-step generalized differential transform method", como visto no modelo Malthusiano. Mas quando aplicamos o método MSGDTM com apenas um passo, conhecido também como "generalized differential transform method"(GDTM), a solução numérica se assemelha como a solução analítica dada em termos da função de Mittag-Leffler. 


\section{Referências}

[1] I. H. Abdel-Halim Hassan. Application to Differential Transformation Method for Solving Systems of Differential Equations. Applied Mathematical Modelling, volume 32 , number 12, pages 2552-2559, 2008.

[2] S. Arshad, A. Sohail, and S. Javed. Dynamical Study of Fractional Order Tumor Model. International Journal of Computational Methods, volume 12, number 5, pages 12,2015 .

[3] R. F. Camargo, and E. C. Oliveira. Cálculo Fracionário. São Paulo: Editora Livraria da Física, 2015. 184p.

[4] B. Ebenezer, K. Awuah-Werekoh, and J. Acquah. Solution of HIV and Malaria Coinfection Model Using Msgdtm. Journal of Mathematics Research, volume 7, number 4, pages 181-193, 2015.

[5] V. S. Erturk, G. Zaman, and S. Momani. A numeric-analytic method for approximating a giving up smoking model containing fractional derivatives. Computers and Mathematics with Applications, 64:3065-3074, 2012.

[6] A. Freihat, and S. Momani. Application of Multistep Generalized Differential Transform Method for the Solutions of the Fractional-Order Chua's System. Discrete Dynamics in Nature and Society, volume 2012, number 427393, pages 12, 2012.

[7] A. H. Handam, and A. A. Freihat. A new analytic numeric method solution for fractional modified epidemiological model for computer viruses. Applications and Applied Mathematics, volume 10, Issue 2, pages 919-936, 2015.

[8] L. K. B. Kuroda. Cálculo Fracionário Aplicado em Dinâmica Tumoral: Método da Transformada Diferencial Generalizada. Botucatu, 2016. pages 105. Dissertação (Mestrado) - Instituto de Biociências, Universidade Estadual Paulista-UNESP.

[9] L. K. B. Kuroda, A. V. Gomes, R. Tavoni, P. F. A. Mancera, N. Varalta, and R. F. Camargo. Unexpected Behavior of Caputo Fractional Derivative. Computational and Applied Mathematics, pages 11, 2015. doi:10.1007/s40314-015-0301-9.

[10] F. Mirzaee. Differential Transform Method for Solving Linear and Nonlinear Systems of Ordinary Differential Equations. Applied Mathematical Sciences, volume 5, number 70, pages 3465-3472, 2011.

[11] Z. Odibat, C. Bertelle, M. Aziz-Alaoui, and G. H. E. Duchamp. A Multi-step Differential Transform Method and Application to Non-chaotic or Chaotic Systems. Computer and mathematics with applications, volume 12, pages 15, 2010.

[12] D. G. Zill, and M. R. Cullen. Equações Diferenciais. Terceira Edição. São Paulo: Pearson Makron Books, 2001. 